\title{
An Efficient Algorithm for Dynamic Bandwidth Allocation
}

\author{
Richard E. Martin and Kenneth M. Guild \\ School of Computer Science and Electronic Engineering \\ The University of Essex \\ Wivenhoe Park \\ Colchester
}

\begin{abstract}
Dynamic networks offer an unprecedented degree of flexibility, enabled by advanced signaling and automation. Concurrently, advances in Deep Packet Inspection (DPI) techniques have made measuring and monitoring more powerful than ever before. In this paper, we propose a novel algorithm to bridge the gap between these disciplines by making networks more autonomous, with automated control of bandwidthprovisioning decisions.
\end{abstract}

\section{INTRODUCTION}

A dynamic network is one in which rapid, simple redistribution of bandwidth has been facilitated by advanced control- and data-plane architectures. The dataplane must support the flexible addition and deletion of bandwidth in a transparent manner with no loss-of-service. The control-plane must offer fast, automated interfaces which allow for signaling between the edge-network devices and the network control to request such bandwidth changes.

Within such dynamic networks, the assignment, redimensioning, and de-assignment of bandwidth provision is simple and fast, with reduced involvement from human operators. Concurrently, measuring and monitoring equipment is more powerful than ever, with SNMP now being reinforced with deep-packet inspection, allowing the identification of flows by application-type [1]. However, at present the bandwidth provision requires human intervention: first, to assess the need (aided by the measuring and monitoring facilities available) and then to use network management to facilitate provision. We seek to automate this process by combining measuring and monitoring with automated signalling, allowing autonomous decisions about network capacity to be made at the edge of the network by suitable traffic-monitoring algorithms.

\section{ABOUt THIS PAPER}

Within this paper, we use an intellectually and computationally simple algorithm that accounts for traffic variability in order to enable the autonomous optimisation of bandwidth provision, a process termed Dynamic Bandwidth Allocation (DBA). We denote this algorithm the DYnamic Linear Bandwidth Estimation through Regression of Traffic (DYLBERT). The performance of this algorithm will be compared with that of the StatisticalDecision (SD) method [2] by assessing the ability of both algorithms to correctly match the observed traffic with their own predictions of bandwidth usage over a single, point-to-point link.

\section{THE TRAFFIC}

The traffic, defining bandwidth demands $b$ measured in Mbps at regular intervals $i$ denoted $b_{i}$, was created using a signal-in-noise model to match the assumptions made in [2]. A sinusoidal function was used to create the "signal" data-set, $s_{i}$.

$$
s_{t}=\left[\sin \left(\frac{t \cdot 2 \cdot \pi}{1440}\right)+2\right] \cdot \frac{2000}{3}, 0<r \leq 525600
$$

In further keeping with the paper [2], we define the noise component $w_{t}$ as i.i.d. Gaussian observations thus:

$$
w_{t} \sim N(\mu, \sigma)
$$

where $\mu$ denotes the mean, and $\sigma$ the standard deviation.

In order to emulate a range of variation, three data-sets were created using a mean of 0 with three values of the standard deviation $(S): 10,25$ and $50 \mathrm{Mbps}$, accordingly:

$$
x_{t}=s_{t}+w_{t}
$$

where $x_{t}$ denotes the traffic utilised in Mbps at time $t$.

\section{The Control Plane}

In order to focus on an evaluation of the performance of the algorithms, the control plane is defined very simply:

1 Determinism: The control plane always responds within the same, constant, time referred to as the delay.

2 Atomic: The control plane will not accept a request to modify bandwidth provision while another is being enacted.

Following from this definition, bandwidth request in interval $i$ will be fulfilled in interval $i+d$; however, during the period of $(i+1, i+2, \ldots i+d)$ the control plane will not accept requests for bandwidth modification. 


\section{The Data Plane}

We consider only symmetric provision where there is sufficient capacity on the terminating interfaces to satisfy any bandwidth requests.

The prediction of a DBA algorithm may be a continuous value, $B, B \in \mathbb{R}^{+}$. As the data-plane is able to process bandwidth requests only in multiples of the data-plane granularity, the bandwidth request $R$ is rounded appropriately using a ceiling function

\section{The DYLBERT Algorithm.}

Within this paper we use a fitted linear model determined using the ordinary least squares method [3] to predict bandwidth requirements. Such a system seeks to fit a function to the data (here referred to as $Y_{i}$ to follow the conventions of linear regression) as defined below:

$$
y_{i}=a+b \cdot x_{i}
$$

where $a$ is the intercept on the $x$ axis, and $b$ is the gradient. $b$ is of most import, as it tells us about the change in traffic demand over time, implying the need for greater or lesser bandwidth provision. The standard deviation of the residuals is defined as:

$$
\sigma_{r}=s d\left(y_{i}-Y_{i}\right), 0<i \leq n
$$

This allows us to compensate for the error inherent in the least-squares fit, which may lead to packet loss.

From the definition of Delay in $\S I V$, it is clear that bandwidth requested in interval $i$ will be fulfilled in interval $i+$ delay. Therefore, the bandwidth requested at $i$ will have to suffice for the interval $i+$ delay to $i+(2 \cdot$ delay $)$. To account for this, the bandwidth requested at interval $i$, denoted $B_{i}$, is defined as follows:

$$
\begin{gathered}
B_{i}=\max \left(a+b \cdot t+3 \cdot \sigma_{r}\right), \quad i+\text { delay }<t<i+(2 \cdot \text { delay }) \\
\text { VII. The SD METhoD }
\end{gathered}
$$

\section{The SD Method}

Recently, a mechanism for bandwidth allocation in TDM networks has been proposed based on specifying thresholds for bandwidth alteration which are linked to the statistical properties of the observed traffic [2], along with the granularity of the data-plane. The purpose of the algorithm is to reduce Unstable Determination, defined $\S \mathrm{IX}-\mathrm{C}$.

Formally, the threshold to increase traffic $\left(T_{1}\right)$ and to decrease traffic $\left(T_{2}\right)$ are linked to the capacity of each TDM link (which they denote $L_{0}$ ), the number of such links already provided $(N)$, and the standard deviation $(\sigma)$ thus:

$$
\begin{aligned}
& T_{1}(N)=N \cdot L_{0}-3 \cdot \sigma \\
& T_{2}(N)=(N-1) \cdot L_{0}-3 \cdot \sigma
\end{aligned}
$$

The algorithm defines a window of the previous 64 datapoints which it uses to determine path addition or deletion. The number of times that the traffic exceeds or falls below a threshold is counted; exceeding the threshold for addition adds to a variable they denote $x$, while falling below the threshold for path deletion adds to a variable they denote $y$ thus:

$$
\begin{aligned}
w & =\left\{x_{(t-63)}, x_{(t-62)} \ldots x_{t}\right\} \\
x & =\left|\left\{x: x \in w, x>T_{1}(N)\right\}\right| \\
y & =\left|\left\{x: x \in w, \quad x<T_{2}(N)\right\}\right|
\end{aligned}
$$

Following on from a thorough mathematical analysis, the authors of the paper define cricital values of $x$ and $y$ which cause path addition and deletion, defining the threshold of $x$ as 20 and $y$ as 44 .

\section{About the Simulator}

The data describing the traffic demands was used as the input to a DBA algorithm simulator implemented in multithreaded ISO C99 which models the control plane and the network interfaces, as well as providing implementations of the algorithms themselves. The SD mechanism was implemented based on the information provided within the original paper [2]. The DYLBERT algorithm builds up the ordinary-least-squares linear-regression functions provided by the GNU Scientific Library [4].

\section{Defining Performance Statistics}

Internally, the simulator generates a vector $B_{t}$, where $B$ indicates the bandwidth assigned at time $t$, having taken into account the effect of the control- and data-plane properties on the requests from the DBA algorithms. By comparing this with $x_{t}$ we can define several metrics which allow for the analysis of the performance of the algorithms.

\section{A. Under-provisioning}

Under-provisioning is indicated by intervals during which the algorithm underestimates demand. In a real network scenario, this may cause packet loss and unacceptable congestion on the link, as well as a violation of the ServiceLevel Agreement (SLA). For this reason, it is worth of study.

We define Underprovisioning, denoted $\hat{U}$, as the sum of all negative values of $B-x$ as a percentage of the total bytes, such that:

$$
\hat{U}=100 .\left(\frac{\sum\left[H\left(B_{i}-x_{i}\right) \cdot\left(B_{i}-x_{i}\right)\right]}{\sum x_{i}}\right)
$$

where $H(x)$ denotes the Heaviside step function [5] and $n$ is the number of intervals.

This definition allows us to study Under-provisioning in relation to a guaranteed service level; for example, the performance of an algorithm in relation to a five-nines SLA is easy to evaluate following this definition, as it corresponds to an Under-provisioning level of $10^{-3}$. 


\section{B. Analysing Over-provisioning}

Over-provisioning is indicated by intervals during which the algorithm overestimates demand. This is less problematic than Under-provisioning; Over-provisioning will have no negative effects on the quality of service percieved by the client; however, an excessive Over-provision indicates that greater bandwidth savings could be made, leading to better network utilisation and therefore efficiency.

Over-provisioning, denoted $\hat{O}$, is defined similarly to Under-provisioning:

$$
\hat{O}=100 .\left(\frac{\sum\left[H\left(x_{i}-B_{i}\right) \cdot\left(B_{i}-x_{i}\right)\right]}{\sum x_{i}}\right)
$$

\section{Unstable Determination}

The metric of unstable determination was proposed in [2], defined as any unnecessary change in bandwidth. For example, an increase in provision followed immediately by a decrease (or the oppsite scenario) is considered "unstable".

We adjust this metric to incorporate delay by defining unstable determination as having occurred when an increase follows a decrease (or the inverse occurs) and the holding time for the associated provision is equal to the delay.

We process $B$ into two vectors, $\hat{B}$ giving the bandwidths (omitting repetitions), and $\hat{H}$ giving the associated holding time. From this, we identify all potentially-unstable allocations thus:

$$
t=\left\{t: t \in \mathbb{N}^{+}, \hat{B}_{t-2}=\hat{B}_{t} \wedge \hat{B}_{t} \neq \hat{B}_{t-1}\right\}
$$

allowing us to define $v$, below, indicating the number of unstable determinations:

$$
v=\mid\left\{x: x \in t,\left(\hat{H}_{x}-\text { delay }\right)=0\right\} \mid
$$

It is more-useful to express unstable determination as a percentage of the total signalling; in such a manner, the percentage of errant signals can be studied independently from the change in the total number of signals sent. Therefore, we define Unstable Determination, $\hat{D}$, as

$$
\hat{D}=100 \cdot \frac{v}{\hat{S}}
$$

where $\hat{S}$ indicates the total number of signals, which can be trivially defined from $B$ - any adjacent values which are not equal $\left(B_{i} \neq B_{i+\text { delay }}\right)$ require signalling. $\hat{S}$ is therefore the number of times this occurs, defined in set builder notation thus:

$$
\hat{S}=\left|\left\{t: t \in \mathbb{N}^{+}, B_{t} \neq B_{t+1}\right\}\right|
$$

\section{Results}

\section{A. Granularity}

The SD mechanism operates by the thresholds outlined in $\S \mathrm{VII}$; therefore, the performance should be affected by granularity $\left(L_{0}\right)$, as well as traffic variance.
We expect DYLBERT to deliver more-deterministic performance. The graph in Fig. 1 plots the performance of the SD and DYLBERT algorithms for the different traffic-variances with varying data-plane granularity. On the Under-provisioning graph (logarithmic $y$-axis), Underprovisioning equivalent to a $99.999 \%$ availability is shown.
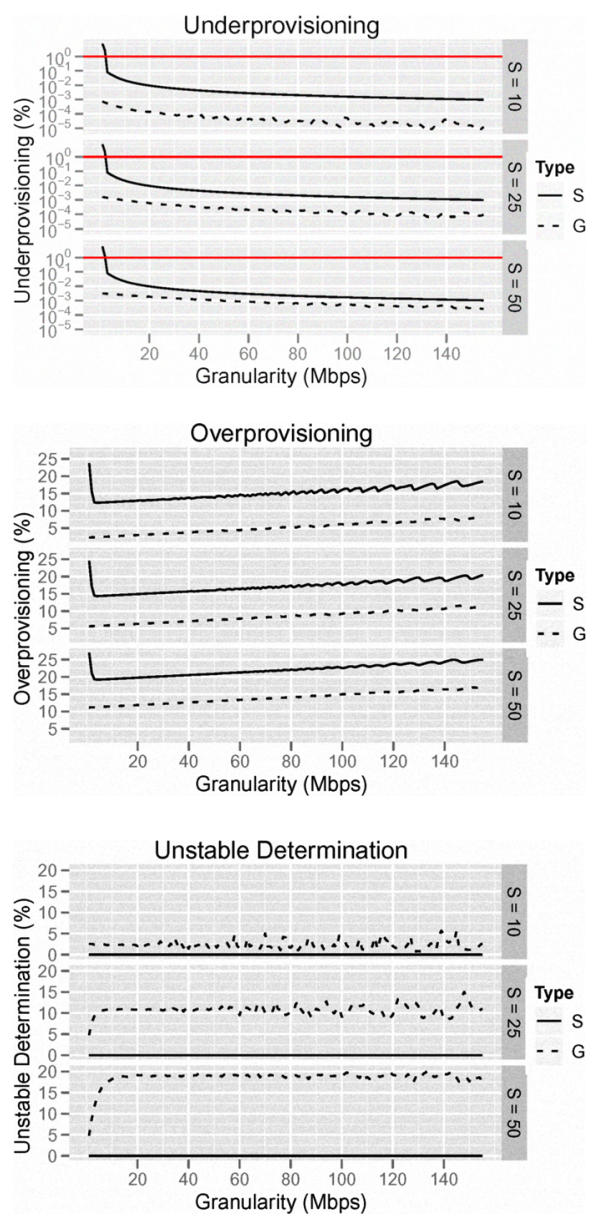

Fig. 1. Graphs showing how the performance of the SD and DYLBERT algorithms vary for three different variances of traffic and with varying data-plane granularity

The aim of the SD algorithm of achieving high determinism has been achieved. This compares favourably with the performance of the DYLBERT algorithm, where Unstable Determination increases significantly with increased traffic-variance, up to almost $20 \%$ in the worst case.

However, it is also clear that the exceptional performance in this area has come at the cost of lower performance in others; the amount of Under-provisioning is almost 100 times higher than DYLBERT in the worstcase, and Over-provisioning is between two and five times higher.

\section{Delay}

The second investigation is into the effect of delay on performance. In order to do so, a data-plane granularity of 20 was chosen on analysis of the data in Fig. 1; the rapid initial decrease in Under-provisioning of the $\mathrm{SD}$ 
algorithm has been overcome by this point, as well as the rapid rise in Unstable Determination of the DYLBERT algorithm. Based on this, the results of varying the control-plane delay can be seen in Fig. 2. Once more, on the Under-provisioning graph (logarithmic $y$-axis), Underprovisioning equivalent to a $99.999 \%$ availability has been highlighted.
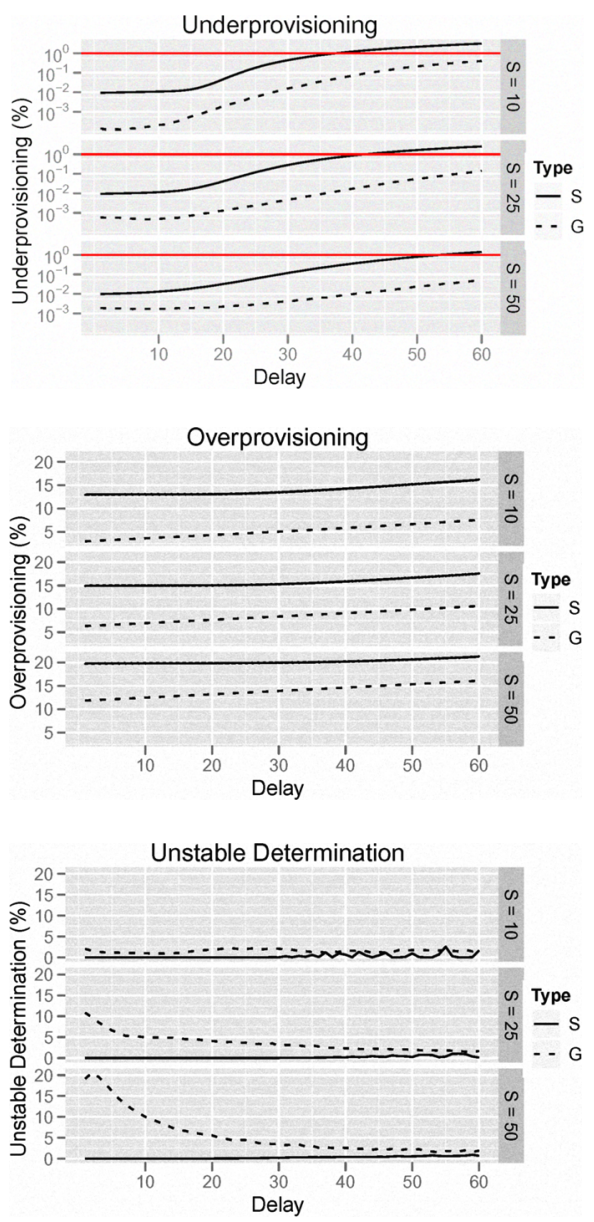

Fig. 2. Graphs showing how the performance of the SD and DYLBERT algorithms vary for three different variances of traffic and with varying control-plane delay

Both algorithms show a clear degradation in performance with increasing delay. This is to be expected; SD no ability to account for delay, while the gradient-based algorithm allows for a limited forecasting with increasing margin-of-error as delay increases. The DYLBERT algorithm maintains its performance advantage over the SD method even as delay increases, showing around 10 times better Under-provisioning performance, and between 10 and $5 \%$ less Under-provisioning.

The SD method maintains its strong performance in the Unstable Determination metric, with only a small percentage of unstable determinations being incurred at high delays.

\section{Discussion}

The purpose of the SD method is to reduce the frequency of unstable determination. While we have shown this, the excellent performance in this area has come at the cost of others. The algorithm suffers from a serious tendency to under and over-provision bandwidth. The DYLBERT algorithm performs much better, resulting in lower Under-provisioning and Over-provisioning, leading to higher utilisation of the provided bandwidth and network equipment. There is, therefore, a trade-off to be had between more-accurate signalling and more-accurate predictions.

From the graphs in Fig. 1, artificially making the granularity more-coarse than the data-plane permits (for example, specifying a granularity of $20 \mathrm{Mbps}$ on an Ethernet network, where the minimum granularity is measured in kilobytes) will markédly decreased signalling traffic while also acting to decrease Under-provisioning, and therefore may make more sense than adopting an algorithm which reduces control plane load at the expense of more-general performance.

\section{CONCLUSION}

Within this paper we have used a novel mechanism for bandwidth allocation within autonomic networks, and compared its performance with the SD algorithm. While we have shown that both algorithms have their advantages and disadvantages, it is clear that for most scenarios the advantages of the SD method do not outweigh its disadvantages of increased Under-provisioning, and Overprovisioning when compared to our algorithm.

\section{ACKNOWLEDGMENT}

This work was funded by the UK Technology Strategy Board (TSB) and Engineering and Physical Sciences Research Council (EPSRC) under the Heterogeneous IP Networks (HIPNet) project (EP/E002382/1). The authors are with the School of Computer Science and Electronic Engineering, University of Essex, Wivenhoe Park, Colchester, U.K. (e-mail: rmarti@essex.ac.uk, kguild@essex.ac.uk).

\section{REFERENCES}

[1] S. Kumar, J. Turner, and J. Williams, "Advanced algorithms for fast and scalable deep packet inspection," in ANCS '06: Proceedings of the 2006 ACM/IEEE symposium on Architecture for networking and communications systems, (New York, NY, USA), pp. 81-92, ACM, 2006.

[2] S. Yamamoto, I. Shake, T. Kurahashi, Y. Tarui, W. Imajuku, Y. Tsukishima, K. Sasayama, O. Moriwaki, and M. Fukutoku, "Theoretical and experimental study of statistical-decision method for link-capacity adjustment," Journal of Lightwave Technology, vol. 25, pp. $2864-2873$, September 2007.

[3] D. Sheskin, Handbook of Parametric and Nonparametric Statistical Procedures. 6000 Broken Sound Parkway NW, Suite 300, Boca Raton, FL 33487-2742: Chapman \& Hall/CRC (Taylor \& Francis Group), fourth ed., 2007.

[4] M. Galassi, J. Davies, J. Theiler, B. Gough, G. Jungman, P. Alken, M. Booth, and F. Rossi, GNU Scientific Library Reference Manual (3rd Ed.). GNU, 2007.

[5] R. Bracewell, The Fourier Transform and its Applications. Boston: McGraw-Hill, third ed., 2000. 